\title{
INFINITELY MANY WEAK SOLUTIONS FOR A FOURTH-ORDER EQUATION WITH NONLINEAR BOUNDARY CONDITIONS
}

\author{
MOHAMMAD REZA HEIDARI TAVANI AND ABDOLLAH NAZARI \\ Received 06 June, 2018
}

\begin{abstract}
Existence results of infinitely many solutions for a fourth-order differential equation are established. This equation depends on two real parameters. The approach is based on an infinitely many critical points theorem.
\end{abstract}

2010 Mathematics Subject Classification: 34B15; 58E05

Keywords: infinitely many solutions, fourth-order equation, critical point theory

\section{INTRODUCTION}

The aim of the present paper is to investigate the existence of infinitely many solutions for the following fourth-order problem

$$
\left\{\begin{array}{l}
u^{(i v)}(x)=\lambda \alpha(x) f(x, u(x))+h(x, u(x)), \quad x \in[0,1], \\
u(0)=u^{\prime}(0)=0 \\
u^{\prime \prime}(1)=0, \quad u^{\prime \prime \prime}(1)=\mu g(u(1))
\end{array}\right.
$$

where $\lambda$ and $\mu$ are positive parameters, $f:[0,1] \times \mathbb{R} \rightarrow \mathbb{R}$ is $L^{1}$-Carathéodory function, $g: \mathbb{R} \rightarrow \mathbb{R}$ is a continuous function , $\alpha \in L^{\infty}([0,1]), \alpha(x) \geq 0$, for a.e. $x \in \mathbb{R}$ ,$\alpha \not \equiv 0$ and $h:[0,1] \times \mathbb{R} \rightarrow \mathbb{R}$ is a Carathéodory function, there exists $0<L<1$, such that $h(x, t) \leq L|t|$ for each $x \in[0,1]$ and $t \in \mathbb{R}$.

The problem (1.1) is related to the deflections of elastic beams based on nonlinear elasticity . In relation with the problem (1.1), there is an interesting physical description.

Suppose an elastic beam of length $d=1$, which is clamped at its left side $x=0$, and resting on a kind of elastic bearing at its right side $x=1$ is given by $\mu g$. Along its length, a load $\lambda \alpha f+h$, is added to cause deformations. If $u=u(x)$ denotes the configuration of the deformed beam, then since $u^{\prime \prime \prime}(1)$ represents the shear force at $x=1$, the condition $u^{\prime \prime \prime}(1)=\mu g(u(1))$ means that the vertical force is equal to $\mu g(u(1))$, which denotes a relation, possibly nonlinear, between the vertical force and the displacement $u(1)$. In addition , since $u^{\prime \prime}(1)=0$ indicates that there is no 
bending moment at $x=1$, the beam is resting on the bearing $\mu g$.

Different models and their applications for problems such as (1.1) can be derived from [4]. We refer the reader to the references [14] and [10] for a physical justification of this model. There is increasing interest in studying fourth-order boundary value problems. Because the change of the static form beam or the support of rigid body can be described by a fourth-order equation. Also a model to study travelling waves in suspension bridges can be furnished by nonlinear fourth-order equations (for instance, see [5]).

When the boundary conditions are nonzero or nonlinear, fourth-order equations can model beams resting on elastic bearings located in their extremities( see for instance $[1,3,6-8,11-13]$ and the references therein).

For example, using a variational methods, the existence of three solutions for special cases of problem (1.1) has been established in [13] and [12]. In [7] the author obtained the existence of at least two positive solutions for the problem

$$
\left\{\begin{array}{l}
u^{(i v)}(x)=f(x, u(x)), \\
u(0)=u^{\prime}(0)=0, \\
u^{\prime \prime}(1)=0, \quad u^{\prime \prime \prime}(1)=g(u(1)),
\end{array}\right.
$$

based on variational methods and maximum principle. It should be noted that the function $f$ is assumed to be continuous. By assuming appropriate conditions on $f$ and $g$, the author guarantees positive solutions to problem (1.2). Also existence and multiplicity results for this kind of problems were considered in $[1,3,6]$. In all these works the critical point theory is applied.

Moreover in [8] authors, considered numerical solutions for problem (1.2) with nonlinear boundary conditions.

In particular, using a variational methods the existence of non-zero solutions for problem

$$
\left\{\begin{array}{l}
u^{(i v)}(x)=f(x, u(x)), \\
u(0)=u^{\prime}(0)=0, \\
u^{\prime \prime}(1)=0, \quad u^{\prime \prime \prime}(1)=\mu g(u(1)),
\end{array}\right.
$$

has been established in [1].

In the present paper, using one kind of infinitely many critical points theorem obtained in [2], we establish the existence of infinitely many weak solutions for the problem (1.1). The paper is organized as follows.

In section 2 we establish all the preliminary results that we need, and in section 3 we present our main results.

\section{PRELIMINARIES}

The following theorem is a smooth version of Theorem 2.1 of [2] which is a more precise version of Ricceri's Variational Principle [9] and it is the main tool of the next section . 
Theorem 1. Let $X$ be a reflexive real Banach space, let $\Phi, \Psi: X \rightarrow \mathbb{R}$ be two Gâteaux differentiable functionals such that $\Phi$ is sequentially weakly lower semicontinuous, strongly continuous, and coercive and $\Psi$ is sequentially weakly upper semicontinuous. For every $r>\inf _{X} \Phi$, let us put

$$
\varphi(r):=\inf _{u \in \Phi^{-1}(]-\infty, r[)} \frac{\sup _{v \in \Phi^{-1}(]-\infty, r[)} \Psi(v)-\Psi(u)}{r-\Phi(u)}
$$

and

Then, one has

$$
\gamma:=\liminf _{r \rightarrow+\infty} \varphi(r), \quad \delta:=\liminf _{r \rightarrow\left(\inf _{X} \Phi\right)^{+}} \varphi(r) .
$$

(a) for every $r>\inf _{X} \Phi$ and every $\left.\lambda \in\right] 0, \frac{1}{\varphi(r)}[$, the restriction of the functional $I_{\lambda}=\Phi-\lambda \Psi$ to $\Phi^{-1}(]-\infty, r[)$ admits a global minimum, which is a critical point (local minimum) of $I_{\lambda}$ in $X$.

(b) If $\gamma<+\infty$ then, for each $\lambda \in] 0, \frac{1}{\gamma}[$, the following alternative holds: either

$\left(b_{1}\right) I_{\lambda}$ possesses a global minimum,

or

$\left(b_{2}\right)$ there is a sequence $\left\{u_{n}\right\}$ of critical points (local minima) of $I_{\lambda}$ such that

$$
\lim _{n \rightarrow+\infty} \Phi\left(u_{n}\right)=+\infty \text {. }
$$

(c) If $\delta<+\infty$ then, for each $\lambda \in] 0, \frac{1}{\delta}[$, the following alternative holds: either

$\left(c_{1}\right)$ there is a global minimum of $\Phi$ which is a local minimum of $I_{\lambda}$, or

$\left(c_{2}\right)$ there is a sequence of pairwise distinct critical points (local minima) of $I_{\lambda}$ which weakly converges to a global minimum of $\Phi$.

Now, we recall some basic facts and introduce the needed notations.

Definition 1. A function $f:[0,1] \times \mathbb{R} \rightarrow \mathbb{R}$ is said to be a Carathéodory function if

$\left(C_{1}\right)$ the function $x \rightarrow f(x, t)$ is measurable for every $t \in \mathbb{R}$;

$\left(C_{2}\right)$ the function $t \rightarrow f(x, t)$ is continuous for a.e. $x \in[0,1]$.

And $f:[0,1] \times \mathbb{R} \rightarrow \mathbb{R}$ is said to be a $L^{1}$-Carathéodory function if, in addition to conditions $\left(C_{1}\right)$ and $\left(C_{2}\right)$, the following condition is also satisfied :

$\left(C_{3}\right)$ for every $\rho>0$ there is function $l_{\rho} \in L^{1}([0,1])$ such that $\sup _{|t| \leq \rho}|f(x, t)| \leq$ $l_{\rho}(x)$ for almost every $x \in[0,1]$.

Denote

$$
X:=\left\{u \in H^{2}([0,1]) \mid u(0)=u^{\prime}(0)=0\right\},
$$


where $H^{2}([0,1])$ is the Sobolev space of all functions $u:[0,1] \rightarrow \mathbb{R}$ such that $u$ and its distributional derivative $u^{\prime}$ are absolutely continuous and $u^{\prime \prime}$ belongs to $L^{2}([0,1])$. $X$ is a Hilbert space with the usual norm

$$
\|u\|_{X}=\left(\int_{0}^{1}\left(\left|u^{\prime \prime}(x)\right|^{2}+\left|u^{\prime}(x)\right|^{2}+|u(x)|^{2}\right) d x\right)^{1 / 2}
$$

which is equivalent to the norm

$$
\|u\|=\left(\int_{0}^{1}\left|u^{\prime \prime}(x)\right|^{2} d x\right)^{1 / 2} .
$$

Also the embedding $X \hookrightarrow C^{1}([0,1])$ is compact and we have

$$
\|u\|_{C^{1}([0,1])}=\max \left\{\|u\|_{\infty},\left\|u^{\prime}\right\|_{\infty}\right\} \leq\|u\|
$$

for each $u \in X$ (see [12]) .

Put

$$
\begin{gathered}
F(x, t)=\int_{0}^{t} f(x, \xi) d \xi \text { for all }(x, t) \in[0,1] \times \mathbb{R}, \\
G(t)=\int_{0}^{t} g(\xi) d \xi \text { for all } t \in \mathbb{R},
\end{gathered}
$$

and

$$
H(x, t)=\int_{0}^{t} h(x, \xi) d \xi \text { for all }(x, t) \in[0,1] \times \mathbb{R} .
$$

Let $\Phi, \Psi: X \rightarrow \mathbb{R}$ be defined by

$$
\Phi(u)=\frac{1}{2} \int_{0}^{1}\left|u^{\prime \prime}(x)\right|^{2} d x-\int_{0}^{1} H(x, u(x)) d x=\frac{1}{2}\|u\|^{2}-\int_{0}^{1} H(x, u(x)) d x
$$

and

$$
\Psi(u)=\int_{0}^{1} \alpha(x) F(x, u(x)) d x-\frac{\mu}{\lambda} G(u(1))
$$

for every $u \in X$.

Now according to (2.1) we observe that

$$
\frac{(1-L)}{2}\|u\|^{2} \leq \Phi(u) \leq \frac{(1+L)}{2}\|u\|^{2}
$$

for every $u \in X$. Similar to [ [1]-page 3], $\Psi$ is a differentiable functional whose differential at the point $u \in X$ is

$$
\Psi^{\prime}(u)(v)=\int_{0}^{1} \alpha(x) f(x, u(x)) v(x) d x-\frac{\mu}{\lambda} g(u(1)) v(1),
$$


and, $\Phi$ is continuously Gâteaux differentiable functional whose differential at the point $u \in X$ is

$$
\Phi^{\prime}(u)(v)=\int_{0}^{1} u^{\prime \prime}(x) v^{\prime \prime}(x) d x-\int_{0}^{1} h(x, u(x)) v(x) d x
$$

for every $v \in X$.

Definition 2. A function $u \in X$ is a weak solution to the problem (1.1) if

$$
\begin{aligned}
\int_{0}^{1} u^{\prime \prime}(x) v^{\prime \prime}(x) d x & -\lambda \int_{0}^{1} \alpha(x) f(x, u(x)) v(x) d x+\mu g(u(1)) v(1) \\
& -\int_{0}^{1} h(x, u(x)) v(x) d x=0
\end{aligned}
$$

for every $v \in X$.

\section{MAIN RESULTS}

Let

$$
\begin{gathered}
G_{\eta}=\min _{|t| \leq \eta} G(t)=\inf _{|t| \leq \eta} G(t) \text { for all } \eta>0, \\
\tau:=\frac{1-L}{8 \pi^{4}\left(\frac{2}{3}\right)^{3}(1+L)}, \\
C:=\liminf _{\xi \rightarrow+\infty} \frac{\int_{0}^{1} \sup _{|t| \leq \xi} \alpha(x) F(x, t) d x}{\xi^{2}}
\end{gathered}
$$

and

$$
D:=\limsup _{\xi \rightarrow+\infty} \frac{\int_{\frac{3}{4}}^{1} \alpha(x) F(x, \xi) d x}{\xi^{2}} .
$$

Now we formulate our main result as follows.

Theorem 2. Assume that

(A1) $\int_{0}^{\frac{3}{4}} \alpha(x) F(x, t) d x \geq 0$ for all $t \geq 0$.

$\left(A_{2}\right) C<\tau D$, where $\tau, C$ and $D$ are given by (3.1), (3.2) and (3.3).

Then, setting

$$
\lambda_{1}:=\frac{4 \pi^{4}\left(\frac{2}{3}\right)^{3}(1+L)}{D}, \quad \lambda_{2}:=\frac{1-L}{2 C},
$$


for each $\lambda \in\left(\lambda_{1}, \lambda_{2}\right)$,for every arbitrary continuous function $g: \mathbb{R} \rightarrow \mathbb{R}$ whose potential

$$
G(t)=\int_{0}^{t} g(\xi) d \xi \quad \text { for all } t \in \mathbb{R},
$$

is a non-positive function satisfying the condition

$$
G^{*}:=-\frac{2}{1-L} \lim _{\xi \rightarrow+\infty} \frac{G_{\xi}}{\xi^{2}}<+\infty
$$

and for every $\mu \in\left(0, \mu_{g, \lambda}\right)$ where $\mu_{g, \lambda}=\frac{1}{G^{*}}\left(1-\frac{\lambda}{\lambda_{2}}\right)$, the problem (1.1) has unbounded sequence of weak solution in $X$.

Proof. From $\left(A_{2}\right)$ we see that $\lambda_{1}<\lambda_{2}$. Our aim is to apply Theorem 1 , to problem (1.1). Fix $\bar{\lambda} \in\left(\lambda_{1}, \lambda_{2}\right)$ and let $g$ be a function satisfies the condition (3.4). Since $\bar{\lambda}<\lambda_{2}$, we have $\mu_{g, \bar{\lambda}}=\frac{1}{G^{*}}\left(1-\frac{\bar{\lambda}}{\lambda_{2}}\right)>0$. Now fix $\bar{\mu} \in\left(0, \mu_{g, \bar{\lambda}}\right)$ and put $z_{1}=\lambda_{1}$ and $z_{2}=\frac{\lambda_{2}}{1+\frac{\mu}{\bar{\lambda}} \lambda_{2} G^{*}}$. If $G^{*}=0$, clearly, $z_{1}=\lambda_{1}, z_{2}=\lambda_{2}$ and $\bar{\lambda} \in\left(z_{1}, z_{2}\right)$. If $G^{*} \neq 0$, since $\bar{\mu}<\mu_{g, \bar{\lambda}}$, we obtain $\frac{\bar{\lambda}}{\lambda_{2}}+\bar{\mu} G^{*}<1$, and so $\frac{\lambda_{2}}{1+\frac{\mu}{\bar{\lambda}} \lambda_{2} G^{*}}>\bar{\lambda}$, namely, $\bar{\lambda}<z_{2}$. Hence, since $\bar{\lambda}>\lambda_{1}=z_{1}$, one has $\bar{\lambda} \in\left(z_{1}, z_{2}\right)$.

Take $X, \Phi$ and $\Psi$ as in the previous section.

For each $u \in X$, we let the functional $I_{\bar{\lambda}}: X \rightarrow \mathbb{R}$ be defined

$$
I_{\bar{\lambda}}(u)=\Phi(u)-\bar{\lambda} \Psi(u) .
$$

$\Phi$ is a sequentially weakly lower semicontinuous on $X$. Indeed, consider an arbitrary $u \in X$ and $\left\{u_{n}\right\}_{n=1}^{\infty} \subset X$ such that $u_{n} \rightarrow u$ in $X$. Due to the compact embedding $X$ into $C([0,1])$, we have that $u_{n} \rightarrow u$ in $C([0,1])$. This implies

$$
\int_{0}^{1} H\left(x, u_{n}(x)\right) d x \rightarrow \int_{0}^{1} H(x, u(x)) d x,
$$

and the weakly sequentially lower semicontinuous property of the $\|$.$\| implies$

$$
\liminf _{n \rightarrow+\infty}\left\|u_{n}\right\|^{2} \geq\|u\|^{2} .
$$

From (3.5)-(3.6) we have

$$
\liminf _{n \rightarrow+\infty} \Phi\left(u_{n}\right) \geq \Phi(u) .
$$

Since

$$
\Phi(u) \geq\left(\frac{1-L}{2}\right)\|u\|^{2},
$$

taking the condition $0<L<1$ into account we observe $\Phi$ is coercive. Moreover, $\Phi$ is strongly continuous. On the other hand, the compact embedding $X$ into $C[0,1]$ 
implies that the functional $\Psi$ is continuously differentiable and with compact derivative. Hence $\Psi$ is sequentially weakly upper semicontinuous (see [15, Corollary 41.9]).Therefore we observe that the regularity assumptions of Theorem 1 on $\Phi$ and $\Psi$ are satisfied . Note that the weak solution of problem (1.1) are exactly the critical points of $I_{\bar{\lambda}}$ (in particular, see [12, Lemma 2.1] ).

Let $\left\{\xi_{n}\right\}$ be a sequence of positive numbers such that $\xi_{n} \rightarrow+\infty$ as $n \rightarrow+\infty$ and

$$
\begin{aligned}
& \lim _{n \rightarrow+\infty}\left(\frac{\int_{0}^{1} \sup _{|t| \leq \xi_{n}} \alpha(x) F(x, t) d x}{\xi_{n}^{2}}-\frac{\bar{\mu}}{\bar{\lambda}} \frac{G_{\xi_{n}}}{\xi_{n}^{2}}\right) \\
= & \liminf _{\xi \rightarrow+\infty}\left(\frac{\int_{0}^{1} \sup _{|t| \leq \xi} \alpha(x) F(x, t) d x}{\xi^{2}}-\frac{\bar{\mu}}{\bar{\lambda}} \frac{G_{\xi}}{\xi^{2}}\right) .
\end{aligned}
$$

According to $\left(A_{2}\right)$ and (3.4), it is obvious that the limit on the right-hand side in (3) is finite and therefore we have

$$
\lim _{n \rightarrow+\infty}\left(\frac{\int_{0}^{1} \sup _{|t| \leq \xi_{n}} \alpha(x) F(x, t) d x}{\xi_{n}^{2}}-\frac{\bar{\mu}}{\bar{\lambda}} \frac{G_{\xi_{n}}}{\xi_{n}^{2}}\right)<+\infty .
$$

Now for all $n \in \mathbb{N}$, put $r_{n}=\left(\frac{1-L}{2}\right) \xi_{n}^{2}$. Since $\left(\frac{1-L}{2}\right)\|u\|^{2} \leq \Phi(u)$, for each $u \in X$ and bearing (2.1) in mind, we see that

$$
\begin{aligned}
\Phi^{-1}(]-\infty, r_{n}[) & =\left\{u \in X ; \Phi(u)<r_{n}\right\} \\
& \subseteq\left\{u \in X ;\left(\frac{1-L}{2}\right)\|u\|^{2}<r_{n}\right\} \\
& \subseteq\left\{u \in X ;|u(x)| \leq \xi_{n} \text { for each } x \in[0,1]\right\} .
\end{aligned}
$$

Note that $\Phi(0)=\Psi(0)=0$. Hence, for all $n \in \mathbb{N}$, one has

$$
\begin{aligned}
\varphi\left(r_{n}\right) & =\inf _{u \in \Phi^{-1}(]-\infty, r_{n}[)} \frac{\sup _{v \in \Phi^{-1}(]-\infty, r_{n}[)} \Psi(v)-\Psi(u)}{r_{n}-\Phi(u)} \\
& \leq \frac{\sup _{v \in \Phi^{-1}(]-\infty, r_{n}[)} \Psi(v)}{r_{n}} \\
& =\frac{\sup _{v \in \Phi^{-1}(]-\infty, r_{n}[)}\left(\int_{0}^{1} \alpha(x) F(x, v(x)) d x-\frac{\bar{\mu}}{\bar{\lambda}} G(v(1))\right)}{r_{n}} \leq
\end{aligned}
$$




$$
\frac{\left(\sup _{v \in \Phi^{-1}(]-\infty, r_{n}[)} \int_{0}^{1} \alpha(x) F(x, v(x)) d x\right)-\frac{\bar{\mu}}{\bar{\lambda}}\left(\inf _{v \in \Phi^{-1}(]-\infty, r_{n}[)} G(v(1))\right)}{\frac{\left(\int_{0}^{1} \sup _{|t| \leq \xi_{n}} \alpha(x) F(x, t) d x\right)-\frac{\bar{\mu}}{\bar{\lambda}}\left(\inf _{|t| \leq \xi_{n}} G(t)\right)}{r_{n}}=}=
$$

Therefore, from (3.8) we obtain

$$
\gamma \leq \liminf _{n \rightarrow+\infty} \varphi\left(r_{n}\right) \leq \lim _{n \rightarrow+\infty} \frac{2}{1-L}\left(\frac{\int_{0}^{1} \sup _{|t| \leq \xi_{n}} \alpha(x) F(x, t) d x}{\xi_{n}^{2}}-\frac{\bar{\mu}}{\bar{\lambda}} \frac{G_{\xi_{n}}}{\xi_{n}^{2}}\right)<+\infty .
$$

Now we show that $\bar{\lambda}<\frac{1}{\gamma}$. Since $\bar{\mu} \in\left(0, \mu_{g, \bar{\lambda}}\right)$ then $\bar{\mu}<\frac{1}{G^{*}}\left(1-\frac{\bar{\lambda}}{\lambda_{2}}\right)=\frac{1}{G^{*}}\left(1-\frac{2 C \bar{\lambda}}{1-L}\right)$. Hence from (3) and (3.9) we have

$$
\begin{gathered}
\gamma \leq \liminf _{n \rightarrow+\infty} \varphi\left(r_{n}\right) \leq \lim _{n \rightarrow+\infty} \frac{2}{1-L}\left(\frac{\int_{0}^{1} \sup _{|t| \leq \xi_{n}} \alpha(x) F(x, t) d x}{\xi_{n}^{2}}-\frac{\bar{\mu}}{\bar{\lambda}} \frac{G_{\xi_{n}}}{\xi_{n}^{2}}\right)= \\
\liminf _{\xi \rightarrow+\infty} \frac{2\left[\int_{0}^{1} \sup _{|t| \leq \xi} \alpha(x) F(x, t) d x-\frac{\bar{\mu}}{\bar{\lambda}} G_{\xi}\right]}{(1-L) \xi^{2}}< \\
\frac{2 C}{1-L}-\lim _{\xi \rightarrow+\infty} \frac{2\left(1-\frac{2 C \bar{\lambda}}{1-L}\right) G_{\xi}}{\bar{\lambda}(1-L) G^{*} \xi^{2}}=\frac{2 C}{1-L}+\frac{1-\frac{2 C \bar{\lambda}}{1-L}}{\bar{\lambda}}=\frac{1}{\bar{\lambda}} .
\end{gathered}
$$

This implies that $\bar{\lambda}<\frac{1}{\gamma}$. So we proved that

$$
\bar{\lambda} \in\left(z_{1}, z_{2}\right) \subseteq\left(\lambda_{1}, \lambda_{2}\right) \subseteq\left(0, \frac{1}{\gamma}\right) .
$$

For the fixed $\bar{\lambda}$, the inequality (3.9) concludes that the condition (b) of Theorem 1 can be applied and either $I_{\bar{\lambda}}$ has a global minimum or there exists a sequence $\left\{u_{n}\right\}$ of weak solutions of the problem (1.1) such that $\lim _{n \rightarrow+\infty}\left\|u_{n}\right\|=+\infty$. 
The other step is to prove that for the fixed $\bar{\lambda}$ the functional $I_{\bar{\lambda}}$ has no global minimum. Let us show that the functional $I_{\bar{\lambda}}$ is unbounded from below. Since $\bar{\lambda}>\lambda_{1}$ then we have

$$
\frac{1}{\bar{\lambda}}<\frac{1}{4 \pi^{4}\left(\frac{2}{3}\right)^{3}(1+L)} \limsup _{\xi \rightarrow+\infty} \frac{\int_{\frac{3}{4}}^{1} \alpha(x) F(x, \xi) d x}{\xi^{2}}
$$

and so there exists a sequence $\left\{\eta_{n}\right\}$ of positive numbers and a constant $\theta$ such that $\eta_{n} \rightarrow \infty$ as $n \rightarrow \infty$ and

$$
\frac{1}{\bar{\lambda}}<\theta<\frac{1}{4 \pi^{4}\left(\frac{2}{3}\right)^{3}(1+L)} \frac{\int_{\frac{3}{4}}^{1} \alpha(x) F\left(x, \eta_{n}\right) d x}{\eta_{n}^{2}}
$$

for each $n \in \mathbb{N}$ large enough. For all $n \in \mathbb{N}$ define

$$
w_{n}(x):= \begin{cases}0 & \text { if } x \in\left[0, \frac{3}{8}\right] \\ \eta_{n} \cos ^{2}\left(\frac{4 \pi x}{3}\right) & \text { if } \left.x \in] \frac{3}{8}, \frac{3}{4}\right] \\ \eta_{n} & \text { if } \left.x \in] \frac{3}{4}, 1\right] .\end{cases}
$$

We observe that $w_{n} \in X$ and $\left\|w_{n}\right\|^{2}=8 \pi^{4}\left(\frac{2}{3}\right)^{3} \eta_{n}^{2}$ and so from (2.4) we see that

$$
\Phi\left(w_{n}\right) \leq \frac{(1+L)}{2}\left\|w_{n}\right\|^{2}=4 \pi^{4}\left(\frac{2}{3}\right)^{3}(1+L) \eta_{n}^{2} .
$$

On the other hand, bearing $\left(A_{1}\right)$ in mind and since $G$ is non-positive, we have

$$
\Psi\left(w_{n}\right) \geq \int_{\frac{3}{4}}^{1} \alpha(x) F\left(x, \eta_{n}\right) d x .
$$

It follows from (3.10)-(3.12) that

$$
\begin{aligned}
I_{\bar{\lambda}}\left(w_{n}\right) & =\Phi\left(w_{n}\right)-\bar{\lambda} \Psi\left(w_{n}\right) \\
& \leq 4(1+L) \pi^{4}\left(\frac{2}{3}\right)^{3} \eta_{n}^{2}-\bar{\lambda} \int_{\frac{3}{4}}^{1} \alpha(x) F\left(x, \eta_{n}\right) d x \\
& <4 \pi^{4}\left(\frac{2}{3}\right)^{3}(1+L) \eta_{n}^{2}-4 \pi^{4}\left(\frac{2}{3}\right)^{3}(1+L) \eta_{n}^{2} \bar{\lambda} \theta= \\
4 \pi^{4}\left(\frac{2}{3}\right)^{3}(1+L) \eta_{n}^{2}(1-\bar{\lambda} \theta) &
\end{aligned}
$$

for every $n \in \mathbb{N}$ large enough. Since $\bar{\lambda} \theta>1$ and $\eta_{n} \rightarrow+\infty$ as $n \rightarrow+\infty$, we have

$$
\lim _{n \rightarrow+\infty} I_{\bar{\lambda}}\left(w_{n}\right)=-\infty
$$


and it follows that $I_{\bar{\lambda}}$ has no global minimum. Therefore, taking the fact

$$
\Phi(u) \leq \frac{1+L}{2}\|u\|^{2}
$$

into account, by $(b)$ from Theorem 1 , there exist a sequence $\left\{u_{n}\right\}$ of critical points of $I_{\bar{\lambda}}$ such that $\lim _{n \rightarrow \infty}\left\|u_{n}\right\|=+\infty$, and the conclusion is achieved.

Now we present the following example to illustrate the result.

Example 1. Let $F:[0,1] \times \mathbb{R} \rightarrow \mathbb{R}$ be the function defined as

$$
F(x, t):= \begin{cases}\mathrm{e}^{\mathrm{x}^{2}} t^{3}(1-\cos (\ln (|t|))) & \text { if }(x, t) \in[0,1] \times(\mathbb{R}-\{0\}) \\ 0 & \text { if }(x, t) \in[0,1] \times\{0\}\end{cases}
$$

and

$$
\alpha(x):= \begin{cases}\frac{1}{4} & \text { if } x \in\left[0, \frac{1}{4}\right] \\ 0 & \text { if } x \in\left(\frac{1}{4}, 1\right]\end{cases}
$$

Consider the problem

$$
\left\{\begin{array}{l}
u^{(i v)}=\lambda \alpha(x) \mathrm{e}^{\mathrm{x}^{2}}\left(3 u^{2}(1-\cos (\ln (|u|)))+u^{2} \sin (\ln (|u|))\right)+\frac{1}{2} \sin u \\
x \in(0,1), \\
u(0)=u^{\prime}(0)=0, \\
u^{\prime \prime}(1)=0, \quad u^{\prime \prime \prime}(1)=\mu g(u(1)) .
\end{array}\right.
$$

Let $f(x, u)=\mathrm{e}^{\mathrm{x}^{2}}\left(3 u^{2}(1-\cos (\ln (|u|)))+u^{2} \sin (\ln (|u|))\right), h(x, u)=\frac{1}{2} \sin u$ with $L=\frac{1}{2}, g(u)=-u$.

We observe that

$$
\begin{gathered}
C:=\liminf _{\xi \rightarrow+\infty} \frac{\int_{0}^{1} \sup _{|t| \leq \xi} \alpha(x) F(x, t) d x}{\xi^{2}}=0 \\
D:=\limsup _{\xi \rightarrow+\infty} \frac{\int_{\frac{3}{4}}^{1} \alpha(x) F(x, \xi) d x}{\xi^{2}}=+\infty
\end{gathered}
$$

and

$$
G^{*}:=-\frac{2}{1-L} \lim _{\xi \rightarrow+\infty} \frac{G_{\xi}}{\xi^{2}}=2 .
$$

Hence, by Theorem 2 , for every $\lambda \in(0,+\infty)$ and $\mu \in\left(0, \frac{1}{2}\right)$ the problem (3.13) has a sequence of generalized solutions which is unbounded in $X$. 
Theorem 3. Assume that assumption $\left(A_{1}\right)$ in Theorem 2 holds and

$$
D>4 \pi^{4}\left(\frac{2}{3}\right)^{3}(1+L) \text { and } C<\frac{1-L}{2}
$$

and for every arbitrary continuous function $g: \mathbb{R} \rightarrow \mathbb{R}$ whose potential $G(t)=\int_{0}^{t} g(\xi) d \xi$ for all $t \in \mathbb{R}$, is a non-positive function, satisfying the condition $\lim _{\xi \rightarrow+\infty} \frac{G_{\xi}}{\xi^{2}}=0$. Then, the problem

$$
\left\{\begin{array}{l}
u^{i v}=f(x, u)+h(x, u), \\
u(0)=u^{\prime}(0)=0, \\
u^{\prime \prime}(1)=0, \quad u^{\prime \prime \prime}(1)=\mu g(u(1))
\end{array} \quad x \in(0,1),\right.
$$

for every $\mu \in(0,+\infty)$ has an unbounded sequence of weak solution in $X$.

Proof. Theorem is an immediately consequence of Theorem 2 when $\lambda=1$.

Remark 1. In Theorem 2, if we assume that the function $f$ is non-negative, the assumption $\left(A_{2}\right)$ can be written as

$$
\liminf _{\xi \rightarrow+\infty} \frac{\int_{0}^{1} \alpha(x) F(x, \xi) d x}{\xi^{2}}<\tau \limsup _{\xi \rightarrow+\infty} \frac{\int_{\frac{3}{4}}^{1} \alpha(x) F(x, \xi) d x}{\xi^{2}}
$$

as well as $\mu_{g, \lambda}=\frac{1}{G^{*}}\left(1-\frac{2 \lambda}{1-L} \liminf _{\xi \rightarrow+\infty} \frac{\int_{0}^{1} \alpha(x) F(x, \xi) d x}{\xi^{2}}\right)$. Moreover, in the autonomous case, putting $F(t)=\int_{0}^{t} f(\xi) d \xi$ for all $t \in \mathbb{R}$, and $\alpha(x)=1$ for a.e. $x \in \mathbb{R}$, the assumption $\left(A_{2}\right)$ assumes the form

$$
\liminf _{\xi \rightarrow+\infty} \frac{F(\xi)}{\xi^{2}}<\frac{\tau}{4} \limsup _{\xi \rightarrow+\infty} \frac{F(\xi)}{\xi^{2}}
$$

and in this case, we have

$$
\lambda_{1}=\frac{16 \pi^{4}\left(\frac{2}{3}\right)^{3}(1+L)}{\lim \sup _{\xi \rightarrow+\infty} \frac{F(\xi)}{\xi^{2}}} \quad \text { and } \quad \lambda_{2}=\frac{1-L}{2 \liminf _{\xi \rightarrow+\infty} \frac{F(\xi)}{\xi^{2}}}
$$

and $\mu_{g, \lambda}=\frac{1}{G^{*}}\left(1-\frac{2 \lambda}{1-L} \liminf _{\xi \rightarrow+\infty} \frac{F(\xi)}{\xi^{2}}\right)$.

Remark 2. Replacing $\xi \rightarrow+\infty$ with $\xi \rightarrow 0^{+}$and also put

$$
C:=\liminf _{\xi \rightarrow 0^{+}} \frac{\int_{0}^{1} \sup _{|t| \leq \xi} \alpha(x) F(x, t) d x}{\xi^{2}}
$$


and

$$
D:=\limsup _{\xi \rightarrow 0^{+}} \frac{\int_{\frac{3}{4}}^{1} \alpha(x) F(x, \xi) d x}{\xi^{2}}
$$

and

$$
G^{*}:=-\frac{2}{1-L} \lim _{\xi \rightarrow 0^{+}} \frac{G_{\xi}}{\xi^{2}}<+\infty
$$

in Theorem 2, by the same way as in the proof of Theorem 2 but using conclusion (c) of Theorem 2 instead of (b), we can obtain a sequence of pairwise distinct weak solutions to the problem (1.1) which converges uniformly to zero.

We present the following example to illustrate Remark 2.

Example 2. Let $\beta>1847$ be a real number and $F: \mathbb{R} \rightarrow \mathbb{R}$ be a function defined by putting

$$
F(t):= \begin{cases}t^{2}\left(1+\beta \sin ^{2}\left(\frac{1}{t}\right)\right) & \text { if } t \in] 0,+\infty[ \\ 0 & \text { if } t \in]-\infty, 0]\end{cases}
$$

We see that $F(t)=\int_{0}^{t} f(\xi) d \xi$, where $f(t)=2 t+2 \beta t \sin ^{2}\left(\frac{1}{t}\right)-\beta \sin \left(\frac{2}{t}\right)$.

Put $g(t)=-t^{3}, \alpha(x)=1$ and $h(x, t)=\frac{1}{3} x t \sin t$ for every $x \in(0,1)$ and $t \in \mathbb{R}$. We can consider $L=\frac{1}{3}$ and in this case $\tau=\frac{27}{128 \pi^{4}}$.

Let $a_{n}=\frac{1}{n \pi}, b_{n}=\frac{1}{\frac{2 n+1}{2} \pi}$. For every $n \in \mathbb{N}$, we have

$$
C:=\liminf _{\xi \rightarrow 0^{+}} \frac{F(\xi)}{\xi^{2}} \leq \lim _{n \rightarrow \infty} \frac{F\left(a_{n}\right)}{a_{n}^{2}}=1,
$$

and

$$
D:=\limsup _{\xi \rightarrow 0^{+}} \frac{F(\xi)}{\xi^{2}} \geqslant \lim _{n \rightarrow \infty} \frac{F\left(b_{n}\right)}{b_{n}^{2}}=\beta+1,
$$

and by (3.18) and (3.19)one has

$$
\liminf _{\xi \rightarrow 0^{+}} \frac{F(\xi)}{\xi^{2}}<\frac{\tau}{4} \limsup _{\xi \rightarrow 0^{+}} \frac{F(\xi)}{\xi^{2}},
$$

as well as

$$
\left(\frac{512 \pi^{4}}{81(\alpha+1)}, \frac{1}{3}\right) \subset\left(\frac{16 \pi^{4}\left(\frac{2}{3}\right)^{3}(1+L)}{\limsup _{\xi \rightarrow 0^{+}} \frac{F(\xi)}{\xi^{2}}}, \frac{1-L}{2 \liminf _{\xi \rightarrow 0^{+}} \frac{F(\xi)}{\xi^{2}}}\right) .
$$


Therefore, by applying Remark 1 and Remark 2, for every $\beta>1847$, and every $\lambda \in\left(\frac{512 \pi^{4}}{81(\alpha+1)}, \frac{1}{3}\right)$ and $\mu \in(0,+\infty)$ the problem

$$
\left\{\begin{array}{l}
u^{i v}=\lambda\left(2 u+2 \beta u \sin ^{2}\left(\frac{1}{u}\right)-\beta \sin \left(\frac{2}{u}\right)\right)+\frac{1}{3} x u \sin u, \quad x \in(0,1), \\
u(0)=u^{\prime}(0)=0, \\
u^{\prime \prime}(1)=0, \quad u^{\prime \prime \prime}(1)=\mu g(u(1))
\end{array}\right.
$$

has a sequence of pairwise distinct weak solutions which converges uniformly to zero.

\section{ACKNOWLEDGEMENT}

The authors are very thankful for the many helpful suggestions and corrections given by the refrees who reviewed this paper.

\section{REFERENCES}

[1] G. Bonanno, A. Chinnì, and S. Tersian, "Existence results for a two point boundary value problem involving a fourth-order equation.” Electron. j. Qual. Theory Differ. Equ., vol. 2015, no. 33, pp. 1-9, 2015, doi: 10.14232/ejqtde.2015.1.33.

[2] G. Bonanno and G. Molica Bisci, "Infinitely many solutions for a boundary value problem with discontinuous nonlinearities." Bound. Value Probl., vol. 2009, pp. 1-20, 2009, doi: $10.1155 / 2009 / 670675$.

[3] M. Grossinhol and S. A.Tersian, “ The dual variational principle and equilibria for a beam resting on a discontinuous nonlinear elastic foundation." Nonlinear Anal., Theory Methods Appl., Ser.A, Theory Methods, vol. 41, no. 3-4, pp. 417-431, 2000, doi: 10.1016/S0362-546X(98)00285-5.

[4] W. W. Jr., S. Timoshenko, and D. Young, Vibrations Problems in Engineering. New York: Wiley, 1990.

[5] A. Lazer and P.J.McKenna, "Large- amplitude periodic oscillations in suspension bridges: Some new connections with nonlinear analysis.” SIAM Rev., vol. 32, no. 4, pp. 537-578, 1990, doi: $10.1137 / 1032120$.

[6] T. F. Ma, "Existence results and numerical solutions for a beam equation with nonlinear boundary conditions." Appl. Numer. Math., vol. 47, no. 2, pp. 189-196, 2003, doi: 10.1016/S01689274(03)00065-5.

[7] T. F. Ma, "Positive solutions for a beam equation on a nonlinear elastic foundation." Math. Comput. Modelling., vol. 39, no. 11-12, pp. 1195-1201, 2004, doi: 10.1016/j.mcm.2004.06.001.

[8] T. F. Ma and J. da Silva, "Iterative solutions for a beam equation with nonlinear boundary conditions of third order." Appl. Math. Comput., vol. 159, no. 1, pp. 11-18, 2004, doi: 10.1016/j.amc.2003.08.088.

[9] B. Ricceri, “ A general variational principle and some of its applications.” J. Comput. Appl. Math., vol. 113, no. 1-2, pp. 401-410, 2000, doi: 10.1016/S0377-0427(99)00269-1.

[10] D. Russel, Mathematical models for the elastic beam and their control- theoretic implications. . Longman, Essex, 1986, pp. 177-216.

[11] X. Wang, "Infinitely many solutions for a fourth-order differential equation on a nonlinear elastic foundation." Boundl. Value Probl., vol. 2013, no. 9, pp. 1-10, 2013, doi: 10.1186/1687-27702013-258. 
[12] L. Yang, H. Chen, and X. Yang, "The multiplicity of solutions for fourth-order equations generated from a boundary condition." Appl. Math. Lett., vol. 24, no. 9, pp. 1599-1603, 2011, doi: 10.1016/j.aml.2011.04.008.

[13] A. Cabada and S. Tersian, "Multiplicity of solutions of a two point boundary value problem for a fourth-order equation." Appl. Math. Comput., vol. 219, no. 10, pp. 5261-5267, 2011, doi: 10.1016/j.amc.2012.11.066.

[14] E. Feireisl, “ Non-zero time periodic solutions to an equation of Petrovsky type with nonlinear boundary conditions: slow oscillations of beams on elastic bearings." Ann. Sc. Norm. Super. Pisa,Cl. Sci., IV. Ser., vol. 20, no. 1, pp. 133-146, 1993.

[15] E. Zeidler, Nonlinear functional analysis and its applications. New York: Springer, 1989.

\section{Authors' addresses}

Mohammad Reza Heidari Tavani

Department of Mathematics,Ramhormoz branch, Islamic Azad University, Ramhormoz, Iran E-mail address: m.reza.h56@gmail.com

\section{Abdollah Nazari}

Department of Mathematics, Kazerun Branch, Islamic Azad University, Kazerun, Iran

E-mail address: nazari_mat@yahoo.com 